\title{
Redescription of Folsomia loftyensis Womersley with notes on the sensillary arrangement of the genital segment in the genus (Collembola: Isotomidae)
}

\author{
Mikhail B. Potapova, Penelope Greenslade ${ }^{\mathrm{b}, \mathrm{c}, \mathrm{d}, *}$ \\ ${ }^{a}$ Moscow Pedagogical State University, Moscow 129164, Kibalchicha St. 6 b. 5, Russia \\ ${ }^{\mathrm{b}}$ Research School of Biology, Australian National University, Australian Capital Territory 0200, Australia \\ ${ }^{\mathrm{c}}$ Environmental Management, School of Science and Engineering, University of Ballarat, Victoria, 3350, Australia \\ ${ }^{\mathrm{d}}$ South Australian Museum, Adelaide, South Australia 5000, Australia
}

Received 12 November 2009; received in revised form 20 January 2010; accepted 20 January 2010

Corresponding Editor: S. De Grave

\section{Introduction}

The genus Folsomia Willem has a cosmopolitan distribution, occurring on all continents. It currently comprises about 150 named species but most species have been described from the Northern Hemisphere. All members of the genus tend to have a reduced number of ocelli, live in leaf litter and soil and some species are parthenogenetic. The genus is poorly represented in

\footnotetext{
Abbreviations: Ant. I-IV, antennal segments I-IV; Abd. I-VI, abdominal segments I-VI; Mac, macroseta; bms, basal microsensillum on antennal segments; ms, microsensillum; PAO, postantennal organ; s, sensillum; Th. II-III, thoracic segments II and III; SAMA, South Australian Museum, Adelaide.

*Corresponding author at University of Ballarat, St Helens, Ballarat, Victoria, 3350, Australia.

E-mail addresses: mpnk@orc.ru (M.B. Potapov),

Pgreenslade@staff.ballarat.edu.au (P. Greenslade).
}

Australia (Greenslade, 1994) and until now the fauna was considered to comprise only a single exotic, introduced species, $F$. candida Willem (Greenslade, 2007). This species has been recorded widely in Australia but only from caves or cultures of animals such as dung beetles. Folsomia similis Bagnall, F. fimetaria (Linnaeus) and $F$ fimetarioides Axelson were also recorded by Womersley (1939) and Greenslade (1994) from Australia but have not been collected recently. Folsomia fimetaroides is likely to be a misidentification.

In our collections we discovered at least two, probably native, Australian species of the Folsomia, one of which was determined as $F$. loftyensis Womersley. This species was transferred to the genus Cryptopygus Willem by Greenslade (1994) because, after studying a number of freshly caught specimens, it appeared that abdominal segments IV and V were separate. However, this was an error and the three last abdominal segments 
are clearly fused. The second species recently collected is probably undescribed but was represented by only a single immature individual, so is not described here.

We provide here a redescription of $F$. loftyensis using topotypical material and record new locations in Australia where it has been found. Morphologically, the species has an unusual form and arrangement of sensilla on fifth abdominal segment, unknown in the genus before. The sensilla of the fifth abdominal segment in other species of Folsomia have already been described (Grow and Christiansen, 1976; Deharveng, 1982; Potapov and Culik, 2002; Potapov and Chimitova, 2009) but a comparison of the different patterns found has not previously been made. In this paper we illustrate the main evolutionary trends in development of these sensillary patterns. About 90 members of the genus, mostly from the Old World, are included in the analysis.

\section{Systematics}

Folsomia loftyensis Womersley, 1934

Type material. Lectotype South Australia, Mt Lofty Ranges, Belair National Park, Long Gully, SAMA I22307, 12.v.1934, leg H. Womersley
Material examined. 5 specimens (incl. adult males and subadult large females): Australia, South Australia, Belair National Park, Long Gully, Karka Oval, moss on timber on ground under eucalypts, $35^{\circ} 01^{\prime}, 139^{\circ} 08^{\prime}$, P.Greenslade 31.v.2009. 7 specimens (incl. subadult females, adult males and juveniles) Australia, Queensland, Lamington National Park, leaf litter, complex Notophyl vine forest, $748 \mathrm{~m},-28.2042 ; 153.1287 \mathrm{E}$, S. Mansell, December, 2008. Victoria, Enfield State Reserve, $10 \mathrm{~km}$ north Dereel, leaf litter from grassy eucalypt woodland, $584 \mathrm{~m}, 37^{\circ} 46.391 \mathrm{E}, 143^{\circ} 46.504 \mathrm{E}$, 12.xii.2009, P. Greenslade.All SAMA.

Description: Body size from $0.6 \mathrm{~mm}$ (small males) to $1.2 \mathrm{~mm}$ (subadult large females). Colour whitish with speckled black pigmentation. Body typical habitus for the genus (Fig. 1). Cuticle with thin hexagonal primary granulation ("smooth"). One ocellus on each side of head, $0.15-0.25$ as long as PAO. PAO narrowly elliptical, constricted, about 1.1 times as long as width of Ant.1 and 1.6-1.7 times length of inner unguis (Fig. 3). Lamellae of maxillary head not enlarged (Fig. 2). Maxillary outer lobe with 4 sublobal hairs, maxillary palp bifurcate. Labral formula as $4 / 5,5,4$. Labium with 5 usual papillae (A-E), full set of guard setae (e7 present), 3 proximal and 4 basomedian setae.

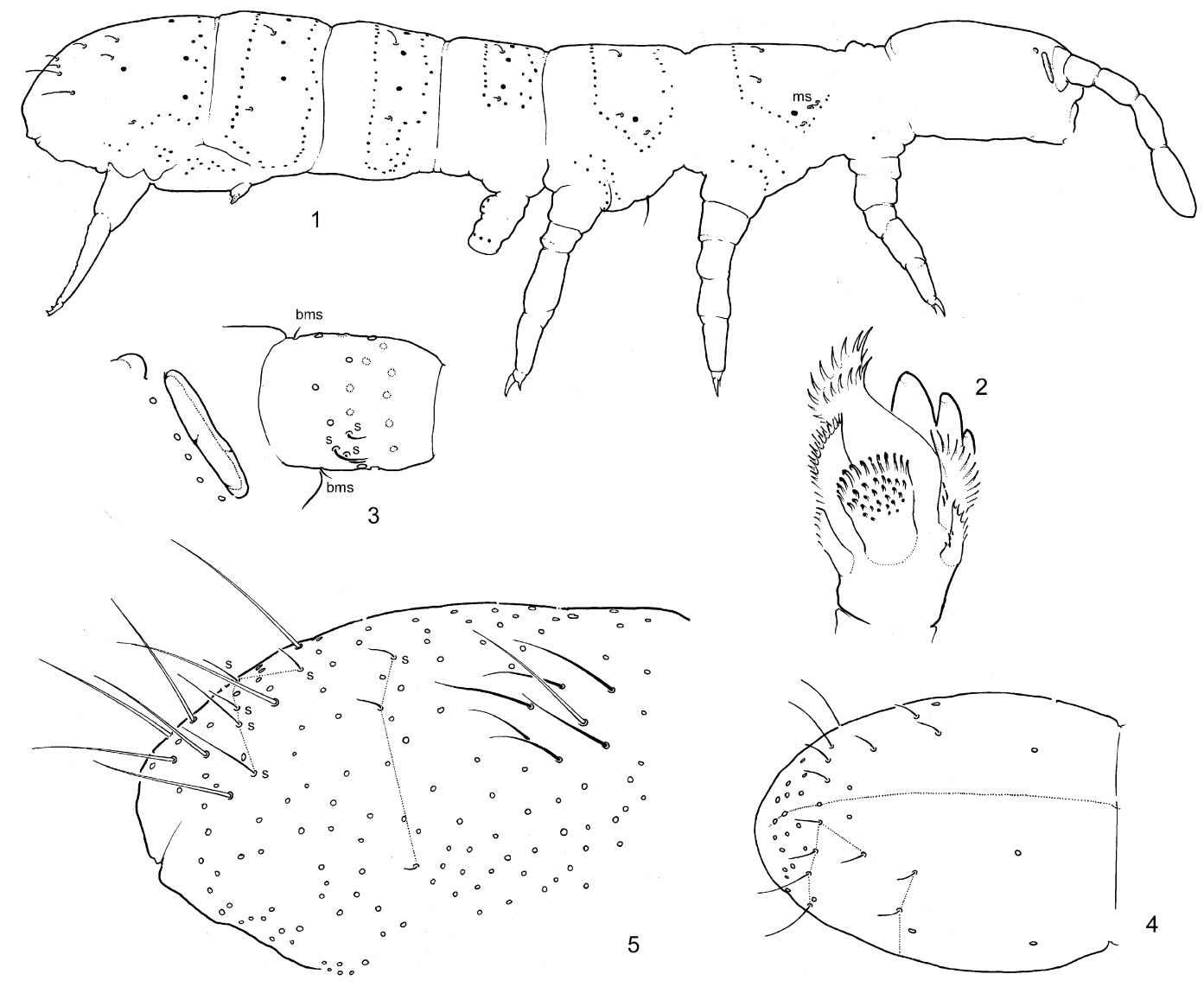

Figs. 1-5. F. loftyensis. 1 - chaetotaxy of abdomen, 2 - maxillary head, 3 - PAO, ocular area and first antennal segment, 4 - arrangement of sensilla and macrosetae on Abd. IV-VI, 5 - chaetotaxy of Abd. IV-VI. 
Ventrally head with $4+4$ postlabial setae. Ant. I with 2 small basal microsensilla (bms), dorsal and ventral, and 3 ventral sensilla (s) (Fig. 3), Ant. II with 3 bms and 1 latero-distal s, Ant. III with large $1 \mathrm{bms}$ and 5 distal s (including 1 lateral). Sensilla on Ant. IV weakly differentiated.

Sensillary formula as 4,3/2,2,2,3,5 (s), 1,0/0,0,0 (ms) (Fig. 1). Tergal sensilla shorter than common setae. Medial sensilla on all tergites situated well anterior of p-row, on Abd. I-III between Mac1 and Mac2. On Abd. $\mathrm{V} 3$ dorsal sensilla nearly equal to sensilla on Abd. IV (Figs. 4, 5). Two lateral sensilla about 2.5 times longer than dorsal ones. Ordinary setae rather long. Macrosetae smooth and long, 1,1/3,3,3 in number, medial ones on Abd. V 1.2-1.3 times shorter than dens and 3.0-3.5 times longer than mucro, hardly differing from ordinary setae. Metathorax with $1+1$ ventral setae. Foil setae at the tip of abdomen absent.

Unguis normal, without lateral and inner teeth. Empodial appendage $0.5-0.6$ as long as unguis. Tibiotarsi I-II always with some additional setae, i.e. more than 25 on each segment. Tibiotarsal tenent setae pointed. Ventral tube with $3+3$ laterodistal and 4-6 posterior setae, anteriorly without setae. Tenaculum with $4+4$ teeth and 1 seta. Anterior furcal subcoxae with 11-12, posterior subcoxa with 5 setae. Anterior side of manubrium normally with from $4+4$ to $5+5$ setae (more rarely with $3+3$ ) of which $3+3$ or $2+2$ arranged in apical transverse row (Figs. 6-8). Posterior side of manubrium with $3+3$ laterobasal setae, about $7+7$ setae medially, lateral setae absent. Dens usually with 12 anterior setae (Figs. 6, 9). Posterior side of dens crenulated and with 6 setae (4 in basal third and 2 medially). Mucro tridentate. Ratio of manubrium:dens:mucro $=2.7-3.5: 3.9-4.3: 1$. Males present.

The specimens from Lamington are darker than those from the type locality and usually have a smaller ocellus. Specimens provisionally identified as Folsomia sp. aff. loftyensis were found on Lord Howe Island, New South Wales (Mt Gower, leaf litter, closed sclerophyl forest, $\left.772-850 \mathrm{~m}, 31^{\circ} 35^{\prime} \mathrm{S}, 159^{\circ} 4^{\prime} \mathrm{E}, 2000-1\right)$. They differed from $F$. loftyensis s.s. in having the sensilla of the lateral duplet of Abd.V thicker and in having only $2+2$ chaetae on anterior side of manubrium (Fig. 6).

\subsection{Comments}

In descriptions of $F$. loftyensis three ocelli on each side were recorded by Womersley $(1934,1939)$. Our examination on the type material of this species has revealed only one ocellus on each side and in his unpublished

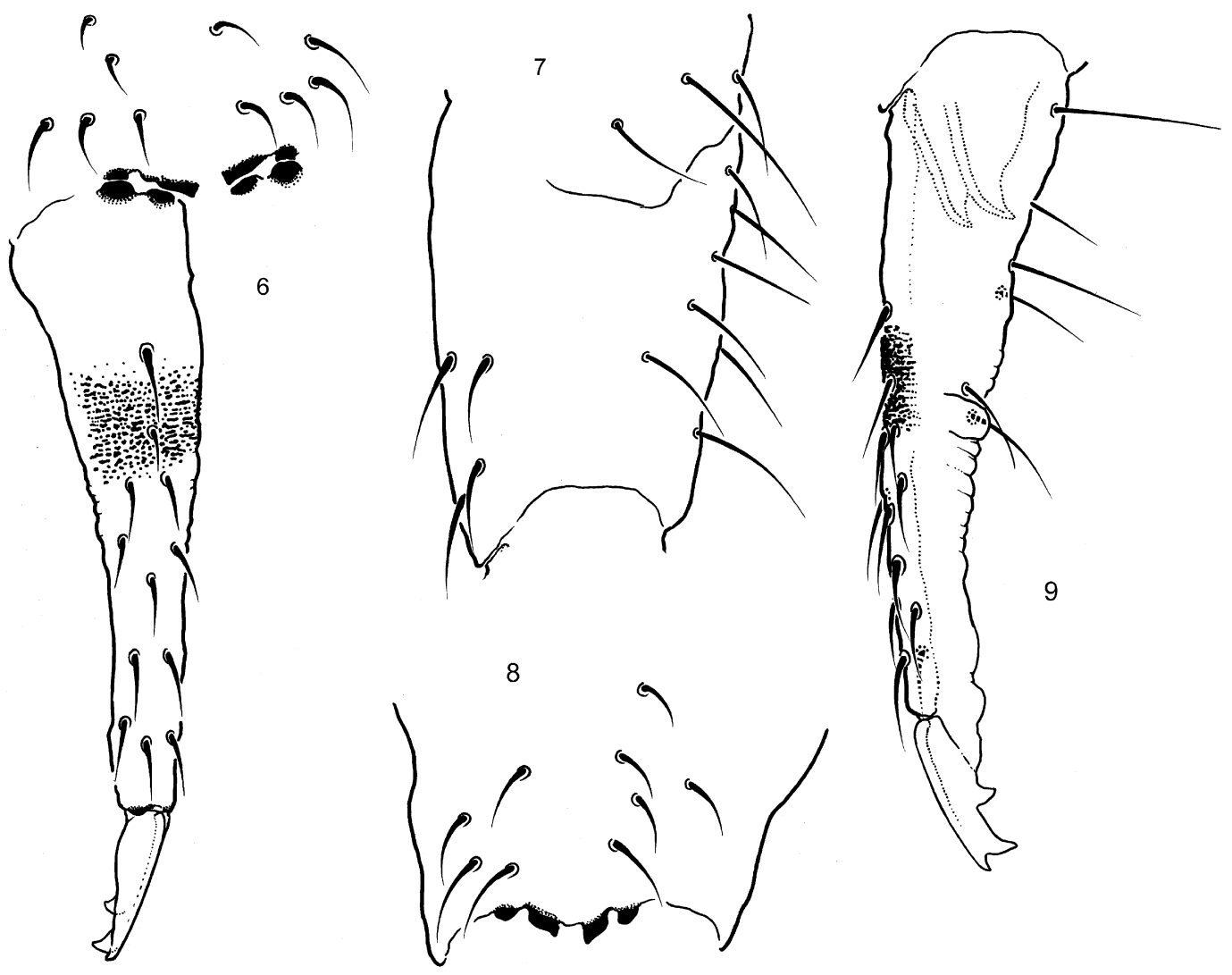

Figs. 6-9. F. loftyensis. 6 - furca and manubrium, anterior view, 7 - manubrium, lateral view, 8 - manubrium, anterior view, another specimen, $\mathbf{9}$ - dens, lateral view. 
revision of Cryptopygus, Peter Lawrence wrote that his "re-examination of the syntypes and of fresh material revealed the presence of no more than a single ommatidium on each side of the head and that the postantennal organ varied from 3 to 6 times its length".

All specimens examined here exhibit well marked rugose chitinouse thickening at the middle of anterior face of the dens (Figs. 6, 9). The feature has been observed in populations of several other species in the genus. As only a few individuals of $F$. loftyensis were seen by us for this study, no decision on the value of this character can be made here. This species shows an unusual pattern of differentiation of sensilla on Abd.V unknown in the genus so far.

\subsection{Distribution and ecology}

The species is quite common in soil, leaf litter, moss and humus in all southern humid regions of Australia and occurs in Tasmania, Victoria, New South Wales, as well as Queensland and South Australia.

\section{Evolutionary trends in development of sensilla on the genital segment in the genus Folsomia}

The genital segment of all species of the genus Folsomia is armed with five sensilla on each side. Following Deharveng (1979) this set is primitive for the whole of the subfamily Anurophorinae s.l. and incorporates four accp-sensilla (accp1, accp2, accp3, accp4) and one as-sensillum ('as' in the text) (Figs. 10,11). This notation system accp-as-al was first proposed by Szeptycki (1972). In later paper by Grow and Christiansen (1976), these sensilla were named ss5, ss6, ss7, ss8 and ss4, respectively and this notation was followed by Fjellberg (2007). In both publications all sensilla of the three last fused abdominal segments, Abd. IV-VI, were considered together and were numbered from ss 1 to ss 8 by Grow \& Christiansen and $\mathrm{s} 1$ to $\mathrm{s} 8$ by Fjellberg. The actual boundary between the segments IV-VI within a conjoint setaceous field can be determined by comparing Folsomia with some species of Proisotoma Börner, where Abd IV-V are not fused, and which have a homologous sensillary pattern (Figs. 10,11). The anterior border of Abd. V is defined as just behind a well-marked transversal row of setae in which two (s2 and s3) of three anterior sensilla are positioned (as a rule, the most medial sensillum s1 is positioned slightly more anteriorly). The posterior border of the segment is considered to be just anterior to the first regular row of posterior macrosetae (labelled fa 1 and fa3 in Figs. 10,11). Posterior to this division, Abd. VI appears to have no sensilla, Abd. V has posterior sensilla s4-s8, which undergo considerable evolutionary changes, and Abd. IV has anterior sensilla s1, s2, s3 (very rarely only s1 and s3) that are rather uniform in shape. This method allowed the sensillary pattern of Abd. V to be determined with certainty for the present paper.

The pattern of the hypothetical ancestor probably consisted of five subequal seta-like sensilla regularly scattered over the whole dorsal and lateral surface of the segment. These sensilla would have been arranged as

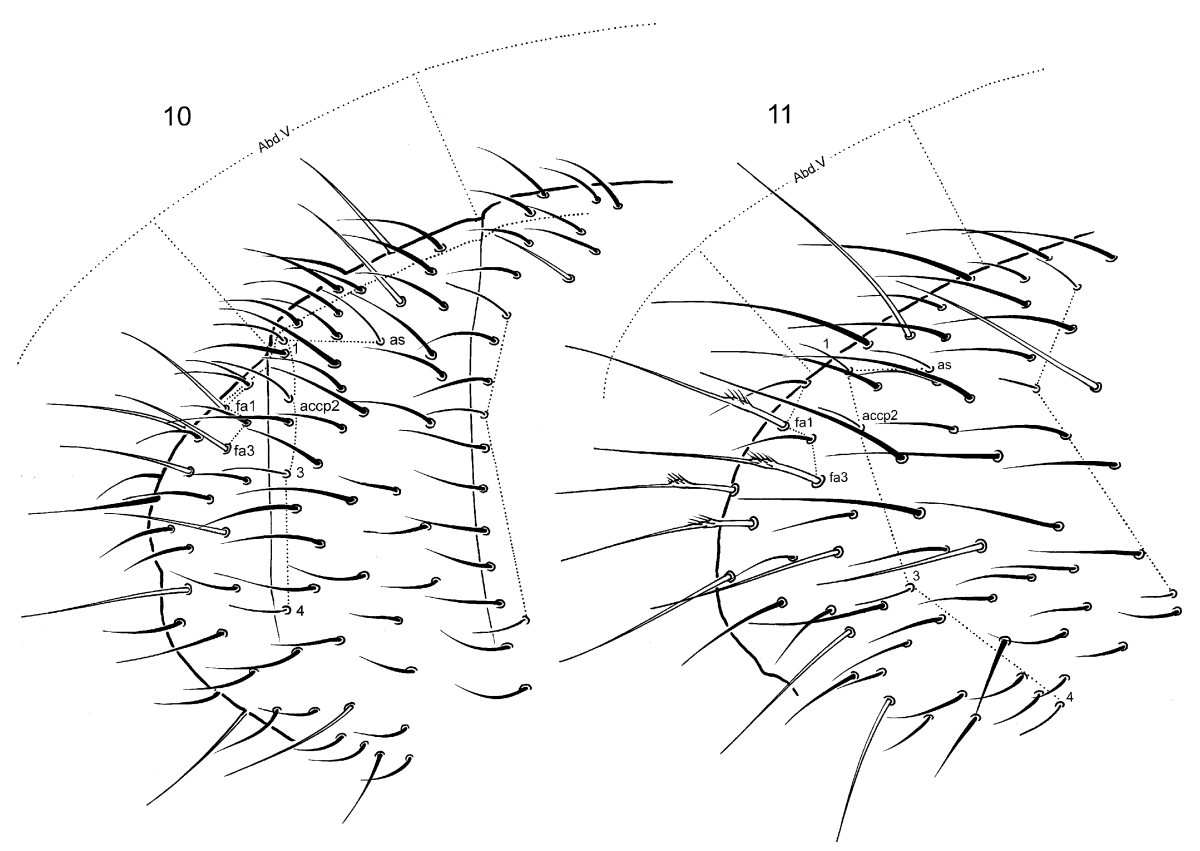

Figs. 10-11. Chaetotaxy of Abd. IV-VI. 10 - Proisotoma (s.1.) sp. (New Zealand), 11 - F. potapovi (Russia: W Siberia). 
four (accp1-4) in an irregular transversal posterior row and one (as) in an anterior-medial position. This pattern is currently found in $F$. potapovi Babenko (Fig. 11). Subsequent evolution varied with the different groups or lineages of species being expressed by a transformation and translocation of sensilla.

The 'fimetaria' s.str. group represents the first step of the translocation in which a migration of accp3 towards the dorsal triplet (as+accp1+accp2) occurs. These four sensilla become grouped more closely together so are more isolated from accp4. This poorly differentiated pattern is found in most species ( $F$. fimetaria L., $F$. candida Willem, F. bisetosa Gisin, F. cryptophila Potapov and Babenko, F. kuznetsovae Potapov and Taskaeva, F. litsteri Bagnall, F. nivalis Packard, F. stella Christiansen and Tucker) (Fig. 16). The length and width of the sensilla remain subequal but they are usually longer than ordinary setae. In two species, (F. sparsosetosa Potapov and Stebaeva, F. stebaevi Potapov), further differentiation occurs. Sensilla of dorsal triplet remain long and the two lateral sensilla (accp3 and accp4) are shortened. In a more extreme case (F. dovrensis Fjellberg) sensilla of the dorsal triplet are dagger-like, accp3 is broadened, and accp4 short. Two species of the 'paoinflata' group ( $F$. paoinflata Potapov, $F$. arena Potapov) and $F$. fimetarioides Axelson ('fimetarioides' group) show a similar pattern, apart from the dagger-like sensilla.

In the 'brevicauda' group four sensilla (as, accp1-3) are grouped together (Fig. 17). The sensilla of the quadruplet formed can be thin ( $F$. bogojevicae Dunger and Zivadinovic), cylindrical ( $F$. brevicauda Agrell), or thick ( $F$. ocellata Jordana) (Fig. 12), and the ventrolateral accp4 sensilla shorter and thinner. This group of species differs in several other uncommon features. A similar pattern was found in F. octooculata Handschin ('alpina' group) with sensilla of the dorsal quadriplet long and thin. Folsomia sp. aff. octooculata (far east of Russia) belonging to the same species group, shows, however, a different pattern (Fig. 16).

In $F$. 'quadrioculata' group s.lato, the fimetaria-like pattern is more extended: sensilla of dorsal triplet are long,

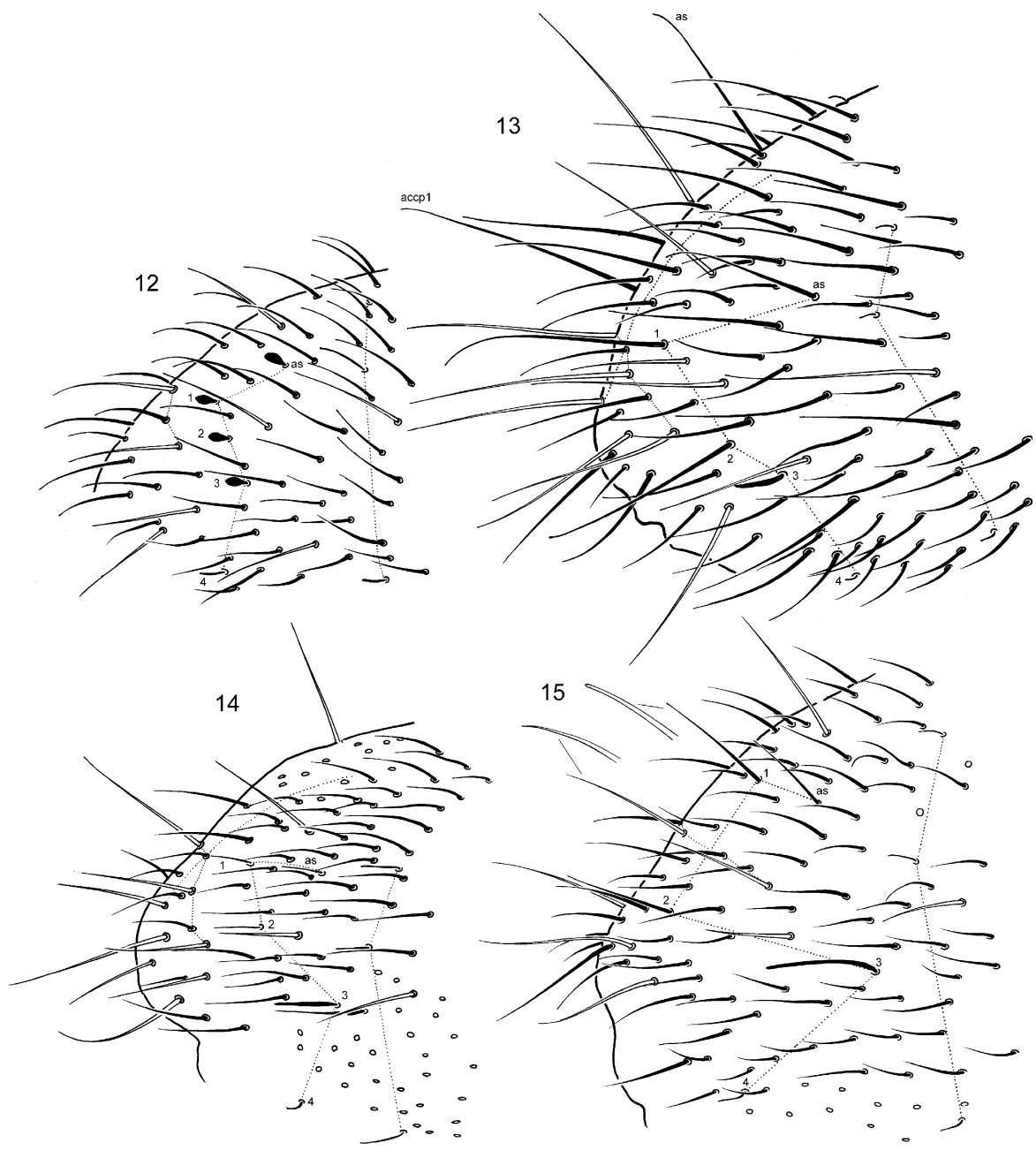

Figs. 12-15. Chaetotaxy of Abd. IV-VI. $12-F$. ocellata (Spain), $13-F$. penicula (Germany), $14-F$. inoculata (Russia: S Siberia), $15-F$. minipunctata (E China). 

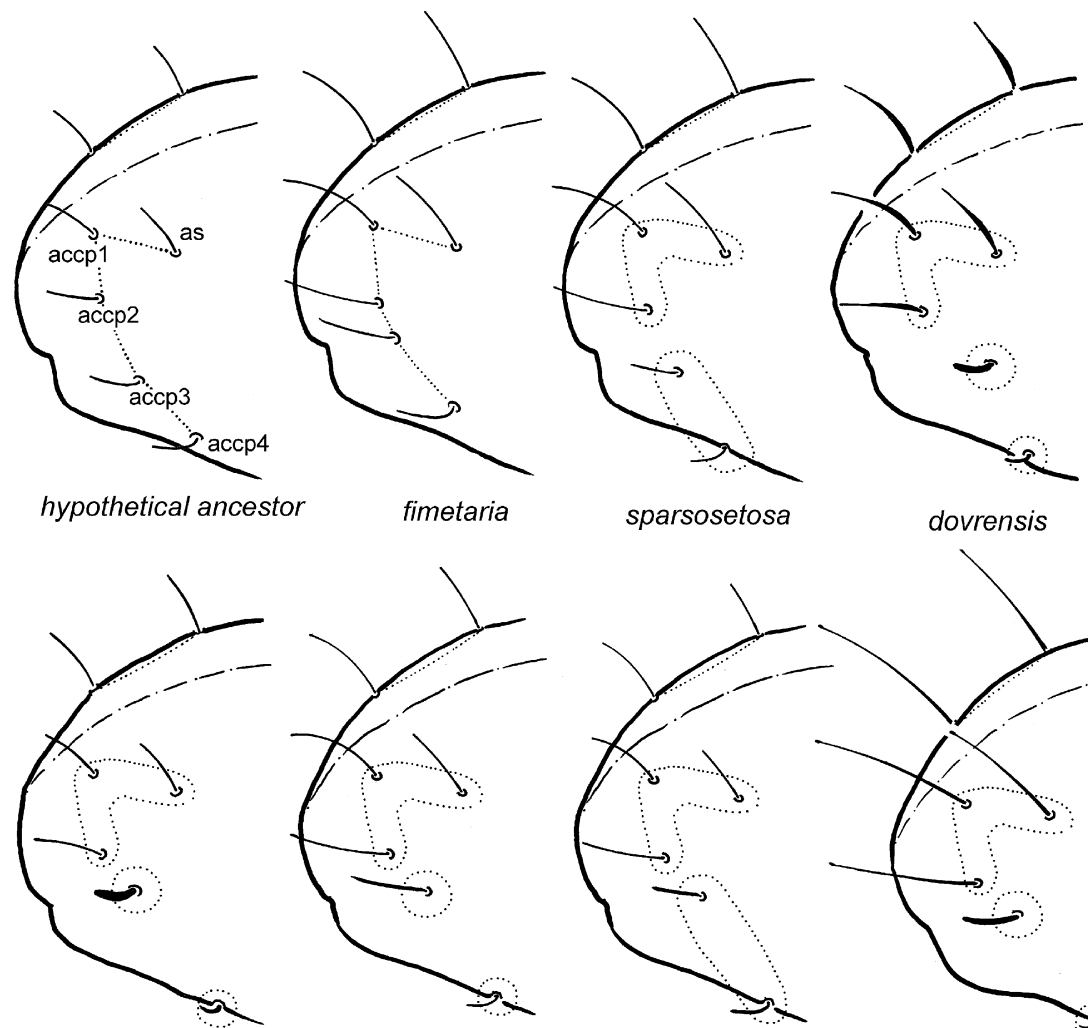

fimetaria

sparsosetosa
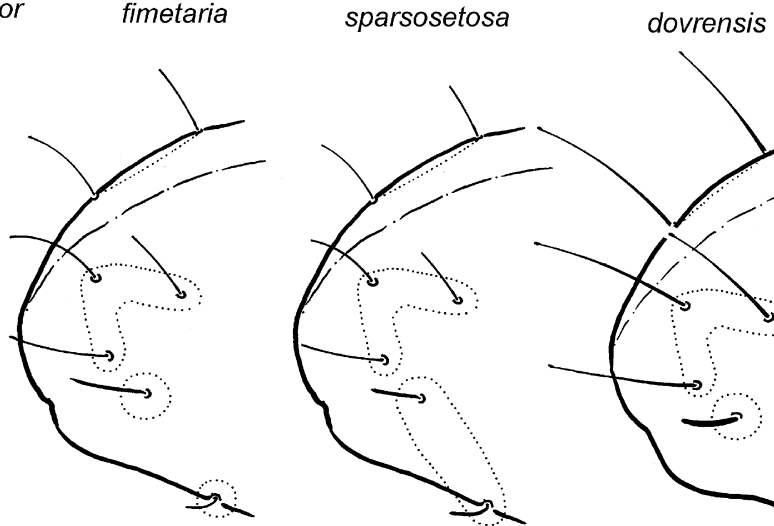

quadrioculata
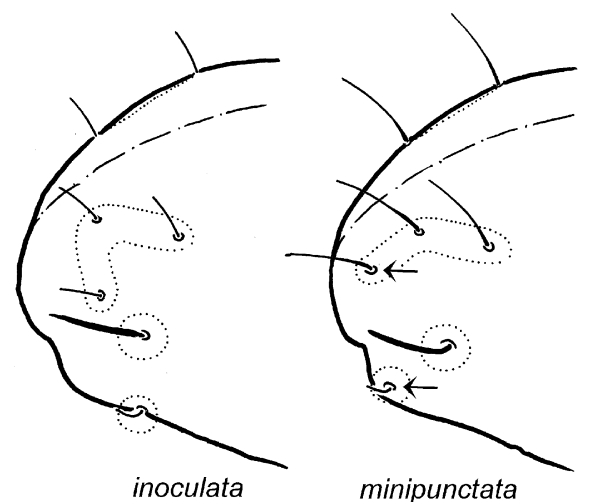

sp, manolachei complex

penicula
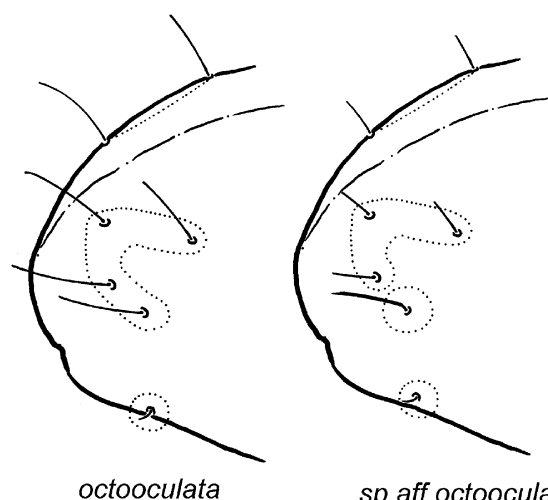

sp.aff.octooculata

Fig. 16. Sensillary patterns of genital segments of Folsomia (hypothetical ancestors, 'fimetaria', 'paoinflata', 'quadriociulata', 'penicula', 'inoculata', 'alpina' groups, and F. minipunctata).

accp 3 is about 1.5 times shorter and 1.5-3.0 times thicker (varying depending on species) and accp4 is short and thin having migrated to a ventrolateral position. This pattern is found in most species $(F$. agrelli Gisin, $F$. albens Kaprus and Potapov, F. amplissima Potapov and Babenko, F. atropolaris Potapov and Babenko, F. baicalica Potapov and Stebaeva, F. binoculata Wahlgren, F. borealis Potapov and Babenko, F. continentalis Potapov and Dunger, $F$. ozeana Yosii, $F$. quadrioculata Tullberg, F. regularis Hammer, F. riozoyoshii Potapov and Cassagnau, F. rossica Potapov and Dunger, F. taimyrica Martynova, F. villosa Potapov and Marusik, F. vtorovi Martynova). In F. palearctica Potapov and Babenko and in some forms of the 'manolachei complex' accp3 and accp4 are subequal. Species of the 'sexoculata' and 'heterocellata' groups show the same pattern as most species of the 'quadrioculata' group but the lateral accp3 sensillum is the same width as the dorsal sensilla.

The 'penicula' group and $F$. inoculata Stach represent a variation of the pattern found in the previous group. In $F$. penicula Bagnall, $F$. spinosa Kseneman, $F$. pseudodiplophthalma Stach and F. taigicola Potapov and Stebaeva ('penicula' group), the sensilla of the dorsal triplet are longer and become macroseta-like (continuously tapering), accp3 thick and rather short and accp4 is small and located dorsoventrally (Fig. 13). 
In contrast, the sensilla of the dorsal triplet of $F$. inoculata are short and accp3 is long (Fig. 14). In most species of the 'inoculata' group ( $F$. baida Potapov, $F$. brevisensilla Potapov and Babenko, $F$. tatarica Martynova, F. torpeda Potapov), the length of al and the four sensilla (as and accp1-3) are very similar, although accp3 is slightly thicker. Folsomia setifrontalis Potapov and Marusik has the more usual pattern with the sensilla of dorsal triplet long. A further evolutionary step is found in F. minipunctata Zhao and Tamura where accp 2 has migrated posteriorly and is located on the last abdominal segment (Fig. 15). This last species has an uncertain taxonomic position and is probably close to species of the 'inoculata' group.

Another line of evolution effects the relative positions of accp3 and accp4 with the latter migrating towards accp 3 and so associated with it. This variation was found in all species of the 'sensibilis' group. Sensilla of the dorsal triplet are long (varying depending on species) and thin and sensilla of lateral duplet (accp3 and accp4) are much shorter and thick (thickness varying according to species) (Fig. 17). Sensilla accp1 often migrates posteriorly and is located on the border between the two last abdominal segments. Considering the position and thickness of accp3 and accp4, F. culter Potapov and Chimitova and F. kurushica Potapov et al. are placed in an intermediate position between the 'sensibilis' group and 'fimetaria' group (Potapov and Chimitova, 2009).

The sensillary pattern of $F$. loftyensis is unlike any previous group (Figs. 4, 5, 17) but has the same arrangement of the three dorsal sensilla forming a triplet and two lateral a duplet as in the sensibilis group.

The most differentiated pattern is expressed by long and thin as-sensillum, short and thin accp1 and accp2, thick accp3 and very short and slightly thickened accp4. It is found in Folsomina onychurina Denis (Fig. 17) that possesses other more apomorphic features compared to Folsomia.

There appear to be no species in which any sensilla on fifth abdominal segment are lost. Records in the literature of the number of sensilla being fewer than five are explained by their sometimes weak differentiation. Sensilla of the dorsal triplet can be very long and macrosetae-like and a small dorsoventral sensilla is usually hardly visible so they were easy to overlook by taxonomists in the past, most frequently in the 'penicula' and 'quadrioculata' groups. In several publications six sensilla were shown for species of the 'sensibilis' group. After examination we conclude that the sixth, ventrally positioned "sensillum" is instead an ordinary, curved, short seta with an unclear homology.
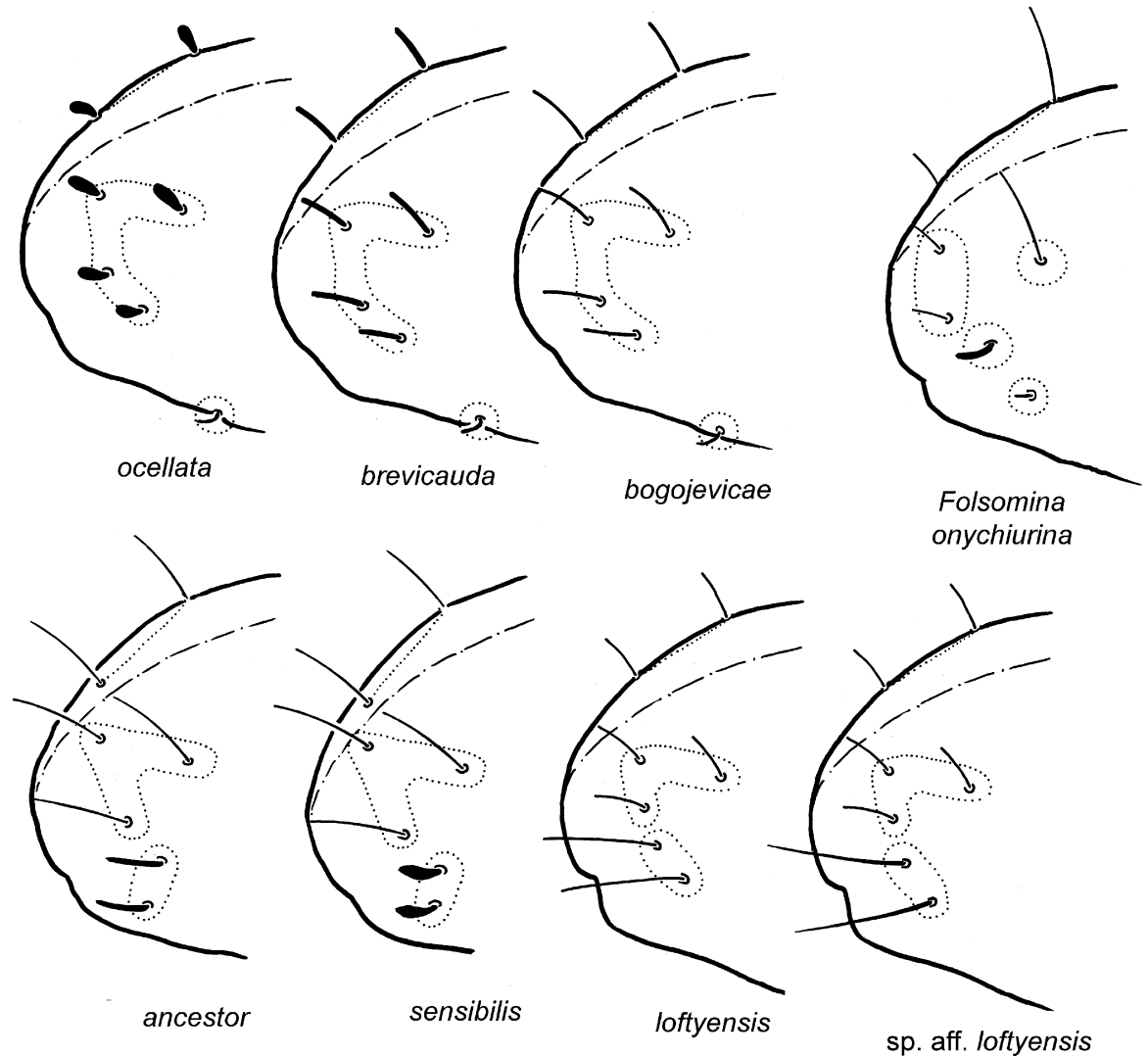

Fig. 17. Sensillary patterns of genital segments of Folsomia ('brevicauda', 'sensibilis' groups, F. loftyensis, F. sp. aff. loftyensis and Folsomina onychiurina). 
Grow and Christiansen (1976) studied nearctic members of the genus and erected six types of chaetotaxy on the three fused abdominal segments. Sensilla, macrosetae, and foil-setae were considered, ventrolateral sensillum accp4 of Abd.V was not considered. Other sensilla of Abd.V were of importance so two types, were erected, called $\mathrm{B}$ and $\mathrm{F}$. Type B was distinguished for $F$. penicula and its North American allies by the very long sensilla of dorsal triplet. This situation was found by us in all species of the 'penicula' group of the Old World and is considered here as an extreme case of differentiation of the " $3+1+1$ " pattern (see below). Type $\mathrm{F}$ was distinguished for Folsomina onychiurina. We also erect the special pattern (" $1+2+1+1$ ") for this species as it exhibits the highest degree of sensillary differentiation. Grow and Christiansen (1976) also used sensillum s3 of Abd. IV (presence/ absence) to characterise their types of chaetotaxy. This sensillum was present in all species studied by us.

\section{Main conclusions}

Following the proposed lines of morphological evolution of springtails proposed by Cassagnau (1980), the main trends in the divergence of patterns and form of sensilla on the genital segment in the genus Folsomia are by means of translocations and especially in transformation. Reduction of number of sensilla does not occur in any of the species studied by us. Translocation is expressed by the migration of the most lateral sensilla accp4 ventrally and the migration of accp 1 or accp 2 posteriorly. Transformation is expressed in differentiation of sensilla in length and width. The method of Nayrolles and Betsch (1993) was partly used to establish transformations and links between setae and groups of setae. The following lines of differentiation are observed:

- as-sensillum and three accp-sensilla (accp1-3) transform as a group with accp4 so forming the " $4+1$ " pattern ('brevicauda' gr.).

- as-sensillum and two accp-sensilla (accp1-2) and accp3 and accp4 form the " $3+2$ " pattern. Sensilla of lateral duplet are short and thick ('sensibilis' group, Holarctic and tropics) or long and slender (F. loftyensis, Australia).

- Dorsal triplet (as and accp1-2), accp3 and accp4 form the " $3+1+1$ " pattern. As a rule, sensilla of dorsal triplet are long and slender, accp3 is mediumsized and thick, and accp4 short and thin (in most of species of the genus). This pattern develops independently in different groups of Folsomia.

- In an extreme case the " $1+2+1+1$ " pattern is developed (Folsomina onychiurina), in which all four groups of sensilla are transformed independently of each other.

\section{Acknowledgements}

We are much indebted to A. Bedos and L. Deharveng (France), A. Babenko (Russia), W. Dunger (Germany), R. Jordana (Spain), L. Yunxia (China), E. Sokolova (Russia) for the loan of valuable material or for collecting samples. We also owe a debt to Peter Lawrence whose unpublished revision was consulted in the course of our work. The study has been partly supported by the Russian Foundation for the Basic Research (GFEN, 0704-92118) and NSFC-RFBR Cooperative Research Project (30811120171).

\section{References}

Cassagnau, P., 1980. Nouveaux critères pour un redécoupage phylogénétique des Collemboles Neanurinae (Massoud, 1976). In: Dallai, R. (Ed.), First International Seminar on Apterygota 115-132.

Deharveng, L., 1979. Chétotaxie sensillaire et phylogènese chez les Collemboles Arthropleona. Trav. Lab. Écobiol. Arthrop. Édaph. 15 (3), 1-15.

Deharveng, L., 1982. A propos des Folsomia du groupe quadrioculata Tullberg, 1871. Rev. Ecol. Biol. Sol. 19 (4), 613-627.

Fjellberg, A., 2007. The Collembola of Fennoscandia and Denmark. Part II: Entomobryomorpha and Symphypleona. Fauna. Entomol. Scand. 42, 1-264.

Greenslade, Penelope, 1994. Collembola. In: Houston, W.W.K. (Ed.), Zoological Catalogue of Australia. vol. 22: Protura, Collembola, Diplura. CSIRO Publishing, Melbourne, pp. 19-138.

Greenslade, Penelope, 2007. The potential of Collembola to act as indicators of landscape stress in Australia. Aust. J. Exp. Agr., 47; 424-434.

Grow, A.B., Christiansen, K., 1976. Chaetotaxy in Folsomia (Collembola Isotomidae) with special reference to Nearctic species. Rev. Ecol. Biol. Sol. 13, 611-627.

Nayrolles, P., Betsch, J.-M., 1993. Pour une théorie de la description chétotaxique chez les Collemboles. Ann. Soc. Entomol. Fr. 29 (1), 5-15.

Potapov, M., Chimitova, A., 2009. Isotomidae (Collembola) of Buryat Republic. Folsomia culter sp. nov and taxonomic value of terminalian chaetotaxy in the genus. Zootaxa 2225, 49-56.

Potapov, M., Culik, M., 2002. A new species of Folsomia (Collembola: Isotomidae) from Brazil, with notes on foilsetae in the fimetaria group. Pan-Pac. Entomol. 78 (2), 69-73.

Szeptycki, A., 1972. Morpho-systematic studies of Collembola. III. Body chaetotaxy in first instars of several genera of Entomobryomorpha. Acta. Zool. Cracov. 17 (15), 341-372.

Womersley, H., 1934. A preliminary account of the Collembola - Arthropleona of Australia. II. - superfamily Entomobryoidea. Trans. Proc Roy. Soc. South Aust. 58, 86-138.

Womersley, H., 1939. Primitive insects of South Australia, Silverfish, Springtails and their Allies. Government Printer, Adelaide. 